\title{
Beyond Fat Mass: Exploring the Role of Adipokines in Rheumatic Diseases
}

\author{
Morena Scotece, ${ }^{1}$ Javier Conde, ${ }^{1}$ Rodolfo Gómez, ${ }^{2}$ Veronica López, ${ }^{1}$ Francisca Lago, ${ }^{3}$ \\ Juan Jesus Gómez-Reino, ${ }^{1}$ and Oreste Gualillo ${ }^{1}$ \\ ${ }^{1}$ Laboratory of Neuroendocrine Interactions in Rheumatology and \\ Inflammatory Diseases (NEIRID LAB), Research Laboratory 9, SERGAS, \\ Institute of Medical Research (IDIS), Santiago University Clinical Hospital, \\ 15706 Santiago de Compostela, Spain \\ ${ }^{2}$ División de Reumatología, Fundación Jimenez Díaz, 28040 Madrid, Spain \\ ${ }^{3}$ Molecular and Cellular Cardiology Laboratory, Research Laboratory 7, SERGAS, \\ Institute of Medical Research (IDIS), Santiago University Clinical Hospital, \\ 15706 Santiago de Compostela, Spain
}

Received 29 July 2011; Accepted 23 September 2011

Academic Editor: Giamila Fantuzzi

The cloning of leptin in 1994 by Zhang et al. introduced a novel concept about white adipose tissue (WAT) as a very dynamic organ that releases a plethora of immune and inflammatory mediators, such as adipokines and cytokines, which are involved in multiple diseases. Actually, adipokines exert potent modulatory actions on target tissues involved in rheumatic diseases including cartilage, synovial, bone and immune cells. The goal of this paper is to elucidate the recent findings concerning the involvement of adipokines in rheumatic diseases, such as rheumatoid arthritis (RA), osteoarthritis (OA), and systemic lupus erythematosus (SLE).

KEYWORDS: white adipose tissue (WAT), adipokines, cytokines, rheumatic diseases, immune response, immune tolerance, metabolism, energetic homeostasis 


\section{INTRODUCTION}

In addition to the central role of lipid storage, white adipose tissue (WAT) is now recognized to be a multifactorial organ. It has a major endocrine function secreting several hormones, most notably leptin and adiponectin, together with a diverse range of other protein signals and factors. These adipose-derived peptides have been termed collectively "adipokines." It is important to underline that these factors might be also synthesized in other tissues, rather than WAT, and participate in other relevant functions correlated with energy homeostasis and metabolism [1].

Adipokines include a variety of proinflammatory peptides. These proinflammatory adipokines are increased in obesity and appear to contribute to the so-called "low-grade inflammatory state" of obese subjects creating a cluster of metabolic aberrations including cardiovascular complications and autoimmune inflammatory diseases.

Initially restricted to metabolic activities, adipokines represent a new family of compounds that can be currently considered as key players of the complex network of soluble mediators involved in the pathophysiology of rheumatic diseases. For instance, obesity has long been considered as a risk factor for osteoarthritis (OA). It has been reported that obesity increases the incidence of OA, particularly in weight-bearing joints such as knees, and weight reduction is correlated with decreased progression of OA. A prevailing hypothesis is that obesity increases mechanical loading across the articular cartilage that leads to its degeneration. However, obesity is also associated with OA in non-weight-bearing joints such as finger joints and wrists, which suggest that these metabolic factors contribute to the high prevalence of OA in obese subjects [2].

This paper addresses current data concerning the involvement of adipokines in the rheumatic diseases, focussing on the role of adipokines played in the pathophysiology of OA, rheumatoid arthritis (RA), and systemic lupus erythematosus (SLE).

\section{LEPTIN AND ADIPONECTIN: A TALE OF TWO GIANTS}

\subsection{Leptin: A Short Overview}

Leptin is the protein product of the $o b$ gene, the murine homologue of the human gene LEP, cloned in 1994 [3]. White adipose tissue cells mainly produce this adipokine, and its plasma concentration is directly correlated with the body-fat stores. It has a central role in fat metabolism; in fact leptin is considered a major regulator of body weight by suppressing appetite and stimulating energy expenditure via hypothalamic receptors. This hormone decreases food intake by inducing anorexigenic factors as cocaine-amphetaminerelated transcript (CART) and increases energy consumption by suppressing orexigenic neuropeptides such as neuropeptide Y (NPY). The biological activity of leptin is mediated by specific receptors (Ob-R), which belong to the class 1 cytokine receptor superfamily and are encoded by the gene diabetes $(d b)$. Alternative splicing of the $d b$ gene produces multiple isoforms, but only the long isoform $\mathrm{Ob}-\mathrm{Rb}$ appears to be capable of transducing the leptin signal.

Leptin is a hormone with pleiotropic actions. In fact, in addition to regulation of food intake, it also affects a variety of other physiological functions, including fertility, bone metabolism, inflammation, infection, and immune responses.

In the last years, important advancements have been added to clarify the involvement of leptin in promoting autoimmune and rheumatic pathologies, particularly rheumatoid arthritis, osteoarthritis, and systemic lupus erythematosus (SLE).

\subsection{Leptin and Osteoarthritis}

It is increasingly evident that this hormone plays a key role in the OA pathophysiology. Leptin expression is much higher in osteoarthritic human cartilage than in normal cartilage, and there exists a strong correlation 
of synovial fluid leptin levels with body mass index (BMI) in people with severe osteoarthritis [4]. The first findings have suggested that high circulating leptin levels in obese individuals may protect cartilage from osteoarthritic degeneration. Actually, Dumond et al. have demonstrated that the intra-articular injection of leptin can strongly stimulate the synthesis of insulin-like growth factor-1 (IGF-1) and transforming growth factor- $\beta$ (TGF- $\beta$ ) at both the messenger RNA (mRNA) and protein levels which can exert anabolic activities in cartilage metabolism [4].

By contrast, leptin has been demonstrated to act as a proinflammatory agent in osteoarthritis. Otero et al. showed that, in cultured human and murine chondrocytes, type 2 nitric oxide synthase (NOS2) is activated by the combination of leptin plus IFN $\gamma$, and NOS2 activation by IL1 is increased by leptin via a mechanism involving JAK2, PI3K, MEK1, and p38 [5-7]. The costimulation of leptin plus IFN $\gamma$ induces nitric oxide, a well-known proinflammatory mediator on joint cartilage, where it triggers chondrocyte phenotype loss, apoptosis, and metalloproteinases (MMPs) activation.

Leptin, per se, is able to induce also the expression of MMPs involved in OA cartilage damage, such as MMP-9 and MMP-13 [8]. Recently, Koskinen et al. have suggested that leptin alone or in combination with IL-1 $\beta$ upregulates MMP-1 and MMP-3 production in human OA cartilage through the transcription factor NF- $\kappa \mathrm{B}$, protein kinase $\mathrm{C}$, and MAP kinase pathways, and its levels correlate positively with MMP-1 and MMP-3 in synovial fluid (SF) from OA patients [9].

Noteworthily, very recently, Gómez et al. have showed that in human chondrocytes leptin increased IL-8 production, which is one of the major mediators of the inflammatory response [10].

Moreover, in articular cartilage of rats, gene expression of ADAMTS-4 and ADAMTS-5 (a disintegrin and metalloproteinase with thrombospondin motifs) was markedly increased after treatment with leptin inducing also a depletion of proteoglycans [11].

Leptin could also contribute to abnormal osteoblast function in OA. Indeed, the elevated production of leptin in OA abnormal subchondral osteoblast is correlated with the increased levels of ALP (alkaline phosphatase), OC (osteocalcin), collagen type I, and TGF- $\beta 1$, inducing a dysregulation of osteoblast function [12]. Very recently, Griffin et al. showed that the incidence of OA was not higher in $o b / o b$ and $d b / d b$ female obese mice than in control background strain (C57BL/6J) [13]. Nevertheless, in this study, no standard was set for the incidence of OA in obese control mice (without leptin mutation) [12].

This recent finding suggests that obesity, as dysregulated body fat accumulation, per se, is not a risk factor for joint degeneration since adiposity in the absence of leptin signaling is insufficient to induce systemic inflammation and knee osteoarthritis in female mice.

\subsection{Leptin and Rheumatoid Arthritis}

Together with other neuroendocrine signals, leptin seems to play a role in autoimmune diseases such as RA, but whether leptin can harm or protect joint structures in RA is still unclear. In patients with RA, circulating leptin levels have been described as either higher or unmodified in comparison to healthy controls $[8,14]$. In RA patients, a fasting-induced fall in circulating leptin is associated with $\mathrm{CD}^{+}{ }^{+}$lymphocyte hyporeactivity and increased IL-4 secretion [15]. Experimental antigen-induced arthritis is less severe in leptin-deficient $o b / o b$ mice than in wild-type mice, whereas leptin-deficient mice and leptin-receptordeficient mice exhibited a delayed resolution of the inflammatory process in zymosan-induced experimental arthritis. Notably, leptin decreased the severity of septic arthritis in wild type mice. So, in the light of the present results it seems difficult to make an unambiguous conclusion about a potential role of leptin in RA [16]. Several authors have also demonstrated that there may exist a close dependence between the risk of aggressive course of RA and leptin levels $[17,18]$. In addition, a correlation between serum leptin and synovial fluid/serum leptin ratio and disease duration and parameters of RA activity has been reported [19].

The action of leptin in RA is not only targeted to articular tissue, but this adipokine also exerts direct modulatory effects on activation, proliferation, maturation, and production of inflammatory mediators in a variety of immune cells, including lymphocytes, natural killer cells, monocytes/macrophages, dentritic cells, neutrophils, and eosinophils [20]. 
In particular, it is known that leptin is able to modulate $\mathrm{T}$ regulatory cells that are potent suppressors of autoimmunity. The group of Matarese has recently demonstrated that leptin secreted by adipocytes sustains Th1 immunity by promoting effector $\mathrm{T}$ cell proliferation and by constraining $\mathrm{T}_{\text {Reg }}$ cells expansion. Weight loss, with concomitant reduction in leptin levels, induces a reduction in effector $\mathrm{T}$ cells proliferation and an increased expansion of $\mathrm{T}_{\text {Reg }}$ cells leading to a downregulation of Th1 immunity and cell-mediated autoimmune diseases associated with increased susceptibility to infections. On the contrary, an increase in adipocyte mass leads to high leptin secretion, which results in expansion of effector $\mathrm{T}$ cells and reduction of $\mathrm{T}_{\mathrm{Reg}}$ cells. This fact determines an overall enhancement of the proinflammatory immunity and of $\mathrm{T}$ cell-mediated autoimmune disorders. Though, leptin can be considered as a link among immune tolerance, metabolic function, and autoimmunity and future strategies aimed at interfering with leptin signaling may represent innovative therapeutic tools for autoimmune disorders.

Very recently it has been demonstrated that leptin can activate mammalian target of rapamycin (mTOR) and regulate the proliferative capacity of regulatory $\mathrm{T}\left(\mathrm{T}_{\mathrm{Reg}}\right)$ cells. This study suggests that the leptin-mTOR signalling pathway is an important link between host energy status and $\mathrm{T}_{\text {Reg }}$ cell activity. Authors conclude that oscillating mTOR activity is necessary for $\mathrm{T}_{\text {Reg }}$ cell activation and suggest that this might explain why $\mathrm{T}_{\text {Reg }}$ cells are unresponsive to TCR stimulation in vitro when high levels of leptin and nutrients may sustain mTOR activation [21, 22]. To note, both direct and indirect effects of leptin on the immune system have been described to account for the immune defects observed in leptin- and leptin-receptor-deficient rodents. Actually, Palmer et al. have also showed an indirect effect of leptin on the immune system, demonstrating that leptin receptor deficiency affects the immune system indirectly via changes in the systemic environment [23].

\subsection{Leptin and Systemic Lupus Erythematosus (SLE)}

Leptin has been suggested to have a role also in other rheumatic diseases such as systemic lupus erythematosus (SLE), in particular modulating the cardiovascular risk of SLE patients. Recently, the group of La Cava demonstrated that leptin and high-fat diet are able to induce proinflammatory high-density lipoproteins and atherosclerosis in BWF1 lupus-prone mice. These data suggest that environmental factors associated with obesity and metabolic syndrome can accelerate atherosclerosis and disease in a lupus-prone background [24].

A relationship between leptin and lupus-disease-related factors is also found. In fact, patients with SLE have increased concentrations of leptin and these concentrations are associated with insulin resistance, BMI (body mass index), and CRP (C-reactive protein) in these patients [25].

\section{ADIPONECTIN}

\subsection{Adiponectin: A Short Overview}

Adiponectin, also known as GBP28, apM1, Acrp30, or AdipoQ, is a 244-residue protein that is produced mainly by WAT. Adiponectin has structural homology with collagens VIII and X and complement factor $\mathrm{C} 1 \mathrm{q}$, and it circulates in the blood in relatively large amounts in different molecular forms (trimers, hexamers, and also 12-18-mer forms) [26, 27]. It increases fatty acid oxidation and reduces the synthesis of glucose in the liver. Ablation of the adiponectin gene has no dramatic effect in knockout mice on a normal diet, but when placed on a high-fat/sucrose diet they develop severe insulin resistance and exhibit lipid accumulation in muscles [28]. Circulating adiponectin levels tend to be low in morbidly obese patients and increase with weight loss and with the use of thiazolidinediones, which enhance sensitivity to insulin [26, 29].

Adiponectin acts via two receptors, one (AdipoR1) found predominantly in skeletal muscle and the other (AdipoR2) in liver. Transduction of the adiponectin signal by AdipoR1 and AdipoR2 involves the activation of AMPK, PPAR- $\alpha$, PPAR- $\gamma$, and other signalling molecules [26]. To note, targeted disruption of AdipoR1 and AdipoR2 causes abrogation of adiponectin binding and all its metabolic actions [30]. 
Actually, some evidences, indicates that adiponectin has a wide range of effects in pathologies with inflammatory component, such as cardiovascular disease, endothelial dysfunction, type 2 diabetes, metabolic syndrome, OA, and RA [31]. Adiponectin acts as a potent modulator of both B and T cells; moreover, it modulates the activity of immune innate response by inducing relevant anti-inflammatory factors such as IL-1 receptor antagonist and IL-10 [26].

\subsection{Adiponectin and RA}

The potential role of adiponectin in rheumatic diseases has been actively investigated. In general, low adiponectin levels have been associated with obesity, type 2 diabetes, atherosclerosis, and vessel inflammation, and in metabolic syndrome the role of adiponectin is clearly anti-inflammatory. On the other side, multiple studies described high adiponectin levels in patients with RA, and these levels correlate with severity of RA $[14,32]$. Giles et al. identified a robust cross-sectional association between serum adiponectin levels and radiographic damage in patients with RA [33], suggesting that this adipokines may be a mediator of the paradoxical relationship between increasing adiposity and protection from radiographic damage in RA, due to adiponectin circulating levels decrease as adiposity increase. Indeed, considering that adiponectin may have negative effects on joint, this adipokine could be a relevant mediator to the inverse relationship between increasing adiposity and radiographic damage observed in RA studies.

In contrast to its "protective" role against obesity and vascular diseases, at joint levels adiponectin might be proinflammatory and involved in matrix degradation. In synovial fibroblasts, adiponectin induces IL-6 production and metalloproteinase-1, two of the main mediators of RA via the p38 MAPK pathway [34]. Similarly, IL-8 is induced by adiponectin through an intracellular pathway involving NF- $\kappa$ B $[10,35]$.

Recent studies showed that adiponectin might also contribute to synovitis and joint destruction in RA by stimulating matrix metalloproteinase-1, matrix metalloproteinase-13, and vascular endothelial growth factor expression in synovial cells, surprisingly, more than conventional proinflammatory mediators (i.e, IL1 beta) [36]. In addition adiponectin increases both cyclooxygenase-2 (COX-2) and membrane-associated PGE synthase-1 (mPGES-1) mRNA and protein expression, in RA synovial fibroblasts (RASFs) in a time- and concentration-dependent manner [37]. This increase was inhibited by siRNA against adiponectin receptor (AdipoR1 and AdipoR2) or using inhibitors of specific proteins involved in adiponectin signal transduction [37].

Recently, Frommer et al. have confirmed the proinflammatory role of adiponectin in RA by demonstrating that this adipokine promotes inflammation through cytokine synthesis, attraction of inflammatory cells to the synovium, and recruitment of prodestructive cells via chemokines, thus promoting matrix destruction at sites of cartilage invasion [38].

\subsection{Adiponectin and $O A$}

It is possible that adiponectin is also implicated in OA pathogenesis. Adiponectin has emerged as a regulator of immune responses and inflammatory arthritis. However, its role in OA and cartilage degradation is controversial and, under many aspects, poorly known. Nevertheless, in chondrocytes this adipokine induces proinflammatory mediators such as nitric oxide, IL-6, MCP-1, MMP-3 and MMP-9 as well as IL-8 [10, 39, 40].

Recent studies show a potential source of adipokines at articular level: the infrapatellar fat pad (IFP). Actually, recent evidence indicates an inflammatory phenotype of this adipose compartment in patients with OA showing that IFP could contribute to the pathophysiological changes in the OA joint via the local production of cytokines and adipokines [41-43]. In addition, the implication of adiponectin in OA pathogenesis is supported also by clinical observations. Lauberg et al. have reported that plasma adiponectin levels were significantly higher in OA patients than in healthy controls [44], and they also observed higher plasma adiponectin levels in female patients with erosive hand OA than those with nonerosive OA [45]. 
It is noteworthy that adiponectin-leptin ratio has been proposed as predictor of pain in OA patients [46]; in fact this adipokine has been detected in the OA synovial fluids correlating with osteoarthritis severity [47] and aggrecan degradation [48].

\subsection{Adiponectin and SLE}

The role of adiponectin in the SLE pathophysiology is not clear. High levels of adiponectin have been found in patients with systemic lupus erythematosus (SLE) in comparison with healthy controls [49]; intriguingly, among the SLE patients, patients with insulin resistance (IR) showed significantly lower adiponectin levels than patients without IR [50].

Rovin et al. have reported that plasma adiponectin levels are increased in patients with renal SLE compared to healthy controls and patients with nonrenal SLE. During renal but not nonrenal SLE flare, urine adiponectin levels increase significantly. For this reason, urine adiponectin may be a biomarker of renal SLE flare [51]. Intriguingly, the group of Aprahamian has suggested that PPAR-gamma agonists may be useful agents for the treatment of SLE and also demonstrated that induction of adiponectin is the major mechanism underlying the immunomodulatory effects of PPAR-gamma agonists [52]. However, these authors obtained their data by using a murine model of lupus so that the reality regarding the potential therapeutic effect of PPAR gamma agonists in human SLE may be completely different.

In addition, the study of Vadacca et al. reported no difference of adiponectin levels in SLE patients in comparison to healthy subjects [53].

In addition, very recently, McMahon and colleagues have demonstrated that leptin levels confer increased risk of atherosclerosis in women with systemic lupus erythematosus and that there is no significant association between adiponectin and atherosclerotic plaques in SLE [24].

\section{RESISTIN}

\subsection{Resistin: A Short Overview}

Resistin, known as adipocyte-secreted factor (ADSF) or found in inflammatory zone 3 (FIZZ3), was discovered in 2001 and was proposed as potential link between obesity and diabetes [54]. It was secreted by adipose tissue but has been found also in macrophages, neutrophils, and other cell types. Serum resistin levels increase with obesity in mice, rats, and humans [55, 56]. Increasing evidence indicates its important regulatory role in various biological processes, including several inflammatory diseases.

\subsection{Resistin and RA}

There are demonstrations that resistin may be involved in the pathogenesis of RA. Increased levels of this adipokine in synovial fluid from patients of rheumatoid arthritis (RA) compared to patients with noninflammatory rheumatic disorders have previously been observed [57].

Actually, resistin has been found in the plasma and synovial fluid of RA patients, and injection of this adipokine into mice joints induce an arthritis-like condition, with leukocyte infiltration of synovial tissues, hypertrophy of the synovial layer, and pannus formation $[58,59]$. Bokarewa et al. have showed also that resistin induces and is induced by several proinflammatory cytokines, such as TNF- $\alpha$ or IL- 6 , in peripheral blood mononuclear cells, via NF- $\kappa$ B pathway, indicating that resistin can increase its own activity by a positive feedback mechanism [58] Increased serum resistin in patients with rheumatoid arthritis correlated with both C-reactive protein (CRP) and DAS28, suggesting a role of this adipokine in the pathogenesis of rheumatoid arthritis [59]. Gonzalez-Gay et al. have confirmed this association between laboratory markers of inflammation, particularly CRP and resistin levels and have showed that anti-TNF-alpha therapy results in a rapid reduction of serum resistin levels in patients with RA [60]. 
There is also an association between resistin and increased inflammation, joint destruction and levels of interleukin 1 receptor antagonist (IL-1RA) in rheumatoid arthritis female patients [61].

\subsection{Resistin and $O A$}

The proinflammatory profile of resistin, together with its association with obesity suggest that this adipokine might be another potential mediator that links OA with inflammation and obesity. It was demonstrated that this adipokine is elevated in both serum and SF after traumatic joint injuries. Recombinant resistin stimulated proteoglycan degradation in mouse femoral head cultures and the induction of inflammatory cytokines and $\mathrm{PGE}_{2}$ production. Moreover, it inhibited proteoglycan synthesis in human cartilage explants [62]. However, Berry et al. have not identified any association between baseline serum levels of resistin and cartilage volume loss [63].

Recently, Zhang and colleagues demonstrated that resistin has diverse effects on gene expression in human chondrocytes, affecting chemokines, cytokines, and matrix gene expression. Messenger RNA stabilization and transcriptional upregulation are also involved in resistin-induced gene expression in human chondrocytes [64].

\subsection{Resistin and SLE}

In addition, resistin has a role as a marker of inflammation in other rheumatic diseases, such as systemic lupus erythematous (SLE). In fact, Almehed et al. have demonstrated a positive correlation between serum resistin levels, inflammation, bone mineral density, and renal functions in patients with SLE [65].

\section{VISFATIN}

\subsection{Visfatin: A Short Overview}

Visfatin, also named pre-B-cell colony-enhancing factor (PBEF) and nicotinamide phosphoribosyltransferase (Nampt), was originally discovered in liver, skeletal muscle, and bone marrow as a growth factor for B-lymphocyte precursors; however, it is also secreted by visceral fat $[66,67]$. It is supposed that visfatin had insulin mimetic properties, but the role of this adipokine in the modulation of glucose metabolism, as well as its binding to insulin receptors, is still amatter of debate [67, 68].

It has been reported that visfatin is increased in obesity. Moreover, leucocytes from obese patients produce higher amounts of visfatin compared with lean subjects, and, specifically, granulocytes and monocytes are the major visfatin-producing cells $[69,70]$. However, leucocytes are not the only nonfat cell type that synthesizes visfatin. Actually, macrophages have been described as a source for visfatin production [71], and, interestingly, this adipokine promoted macrophage survival by reducing apoptosis [72].

\subsection{Visfatin and RA}

Visfatin may be considered another potential therapeutic target for RA with important proinflammatory and catabolic roles in RA pathogenesis. Our group demonstrated that circulating visfatin is higher in patients with RA than in healthy controls [14]. These data were also further confirmed by other authors [73]. To note, enhanced visfatin levels are associated with augmented joint damage [73]. Brentano and colleagues reported that visfatin was localized in the site of invasion of synovial tissue in joints of RA patients. Moreover, it is able to induce IL-6, MMP-1, and MMP-3 in RA synovial fibroblasts, as well as IL-6 and TNF- $\alpha$ in monocytes [74]. To note, PBEF knockdown in RASFs significantly inhibited basal and TLR ligand-induced production of IL-6, IL-8, MMP-1, and MMP-3 [74].

Very recently, Busso et al. have showed that visfatin is a key mediator in inflammatory arthritis. 
The administration of a visfatin inhibitor to mice with collagen-induced arthritis reduced arthritis severity with similar effect to that produced by TNF- $\alpha$ inhibitor [75]. Moreover, pharmacological inhibition of visfatin led to reduced levels of intracellular NAD in inflammatory cells and decreased the production of TNF- $\alpha$ and IL-6 in affected joints [75]. However, the mechanisms by which visfatin exerts its catabolic effect in arthritic joints are still incompletely understood.

\subsection{Visfatin and $O A$}

At cartilage level, OA chondrocytes are able to produce visfatin and its expression is increased after IL$1 \beta$ treatment [76]. Visfatin administration, like IL- $1 \beta$, enhances $\mathrm{PGE}_{2}$ release. In line with this, visfatin also increases MMP-3 and MMP-13 synthesis and release and ADAMTS-4 and ADAMTS-5 expression in mouse articular chondrocytes [76]. Probably due to this augment in the expression of matrix degradative enzymes, visfatin decreases aggrecan expression [76].

In addition, we showed that serum visfatin concentrations were higher in patients with OA compared to healthy controls [11]. Very recently, Duan et al. have reported that SF visfatin was positively correlated with degradation biomarker of collagen II, helix-II, and C-telopeptide of type II collagen (CTX-II) and degradation biomarker of aggrecan, aggrecanase-1 (AGG1), and aggrecanase-2 (AGG2), suggesting an involvement of adiponectin in cartilage matrix degradation [77].

Taken together, these data suggest that visfatin has a catabolic function in cartilage and may have an important role in the pathophysiology of osteoarthritis.

\subsection{Visfatin and SLE}

Recent findings report also an implication of visfatin in SLE pathophysiology. It was showed that, in SLE patients, visfatin levels were higher compared to healthy controls $[49,73]$. However, further studies are needed for more precise elucidation of the role that this adipokine plays in the SLE.

\section{CHEMERIN}

Chemerin, also known as tazarotene-induced gene 2 and retinoic acid receptor responder 2 (RARRES2), is a novel identified chemoattractant adipokine [78]. It is secreted as an $18 \mathrm{kDa}$ inactive proprotein and activated by posttranslational C-terminal cleavage [79]. Chemerin acts via the G-coupled receptor chemokine-like receptor 1 (CMKLR1 or ChemR23) [78]. Chemerin and its receptor are mainly expressed, but not exclusively, in adipose tissue [80], for instance, dendritic cells, and macrophages express chemerin receptor [81]. ChemR23 is also expressed by endothelial cells, and it is upregulated by proinflammatory cytokines such as TNF- $\alpha$, IL-1 $\beta$, and IL-6 [82]. Moreover, chemerin exogenous challenge promotes in vitro angiogenesis by inducing cell proliferation, endothelial migration, and capillary tube formation, critical steps in the development of angiogenesis [82].

Interestingly, chondrocytes express chemerin and its receptor [83-85], and IL-1 $\beta$ is able to increase chemerin expression [84]. In the same way, Berg et al. have demonstrated that recombinant chemerin enhances the production of several proinflammatory cytokines (TNF- $\alpha$, IL-1 $\beta$, IL-6, and IL-8), as well as different MMPs (MMP-1, MMP-2, MMP-3, MMP 8, and MMP-13) in human articular chondrocytes [83]. These factors play a role in the degradation of the extracellular matrix, by causing a breakdown of the collagen and aggrecan framework, and result in the irreversible destruction of the cartilage in OA and RA. Moreover, these authors reported that the intracellular signalling after ChemR23 activation occurs through p42/44 MAPK and Akt phosphorylation.

Chemerin and ChemR23 expression was found in SLE skin biopsies [85]. In vitro experiments showed that chemerin acts as a chemotactic factor for plasmacytoid DCs. The tissue distribution of this adipokine, located at the luminal side of inflamed blood vessels, suggests that chemerin is involved in 
the migration of plasmacytoid DCs and the accumulation of these cells in inflamed tissues in SLE patients [85]. Moreover, De Palma et al. found chemerin expression in renal tubular epithelial cells from SLE patients with nephritis [86]. These authors, using a transendothelial chemotaxis assay, demonstrated that the recruitment of plasmacytoid DCs by TNF- $\alpha$ was mediated by chemerin/ChemR23 interaction, which may be due to the induction of the cleavage of prochemerin by TNF- $\alpha$ through the local production of serine proteases in proximal tubular epithelial cells [79, 86-88].

\section{LIPOCALIN 2}

Lipocalin 2 (LCN2), also termed siderocalin, 24p3, uterocalin, and neutrophil gelatinase-associated lipocalin (NGAL), is a $25 \mathrm{kDa}$ glycoprotein isolated from neutrophil granules although white adipose tissue (WAT) is thought to be the main source [89]. The LCN2 protein has been isolated as a $25 \mathrm{kDa}$ monomer, as a $46 \mathrm{kDa}$ homodimer, and in a covalent complex with MMP-9, and its cellular receptor, megalin (GP330), was recently described [90]. LCN2 is involved in apoptosis of haematopoietic cells [90], transport of fatty acids and iron [91], modulation of inflammation [92], among other processes.

LCN2 has recently been identified in chondrocytes [93]. In these cells IL-1 $\beta$, leptin, adiponectin, LPS, and dexamethasone act as potent modulators of LCN2 expression [84]. Lipocalin 2 is likely to be involved in matrix degradation since it forms molecular complexes with MMP-9 [94].

Recently, the group of Katano confirmed that the level of NGAL in SF was significantly higher in patients with RA than in those with osteoarthritis. Through proteome analysis Katano et al. have showed that GM-CSF may contribute to the pathogenesis of RA by the upregulation of LCN2 in neutrophils, followed by induction of Cathepsin D, transitional endoplasmic reticulum ATPase (TERA), and transglutaminase $2(\operatorname{tg} 2)$ in synoviocytes [35]. These enzymes may contribute to the proliferation of synovial cells and infiltration of inflammatory cells inside the synovia [35].

Finally, LCN2 is also a candidate biomarker for the early detection of LN (lupus nephritis) that is an inflammation of the kidney caused by systemic lupus erythematosus (SLE), which is very common in childhood-onset SLE (cSLE). Hinze et al. have demonstrated that urinary and plasma NGAL (U-NGAL and P-NGAL) are excellent candidates for predictive biomarkers for worsening of cSLE renal and global disease activity, respectively [95].

\section{SERUM AMYLOID A3}

Serum amyloid A3 (SAA3) protein is an adipokine that belongs to the family of acute-phase serum amyloid A proteins (A-SAA) secreted in the acute phase of inflammation. In mice, all A-SAA proteins are actively transcribed [96-98] whereas, in humans, SAA3 is encoded by a pseudogene and its functional protein is unknown $[99,100]$. In contrast to that, in other species, murine SAA3 expression is not confined to the liver but found in several cell types [101-103]. Murine SAA3 is involved in immune, metabolic, and cardiovascular homeostasis [103-105]. Certain factors (e.g., IL-1 $\beta$, TNF, dexamethasone, IL-6, and bacterial LPS) and conditions such as obesity modulate SAA3 expression [101-103, 106]. SAA3 is induced by IL- $1 \beta$ in primary rabbit chondrocytes and can induce transcription of MMP-13 [107].

\section{OTHER ADIPOKINES WITH A POTENTIAL ROLE IN RHEUMATIC DISEASES}

\subsection{Apelin, Vaspin, and Omentin}

\subsubsection{Apelin}

Apelin is a bioactive peptide that was originally identified as the endogenous ligand of the orphan G proteincoupled receptor APJ [108]. TNF increases both apelin productions in adipose tissue and blood plasma 
apelin levels when administered to mice [109]. Intriguingly, in mice with diet-induced obesity, macrophage counts and the levels of proinflammatory agents such as TNF rise progressively in adipose tissue [110]. Thus, one can envisage that overproduction of apelin in the obese might be an adaptive response that attempts to forestall the onset of obesity-related disorders such as mild chronic inflammation.

Very recently, Hu et al. have suggested that apelin may play a catabolic role in cartilage metabolism and that it can be a risk factor in the pathophysiology of osteoarthritis. Apelin stimulates the proliferation of chondrocytes and significantly increases mRNA levels of MMP-1, MMP-3, MMP-9, and IL-1 $\beta$ in vitro. Intra-articular injection with apelin in vivo upregulates the expression of MMP-3, MMP-9, and IL-1 $\beta$ decreases the level of collagen II. In addition, after treatment with apelin, mRNA levels of ADAMTS-4 and ADAMTS-5 markedly increased and depletion of proteoglycan in articular cartilage was found [11].

\subsubsection{Vaspin}

Vaspin is a serpin (serine protease inhibitor) that was produced in the visceral adipose tissue [111]. Interestingly, administration of vaspin to obese mice improved glucose tolerance and insulin sensitivity and reversed altered expression of genes that might promote insulin resistance. The induction of vaspin by adipose tissue might constitute a compensatory mechanism in response to obesity and its inflammatory complications.

\subsubsection{Omentin}

Omentin is a protein of $40 \mathrm{kDa}$ secreted by omental adipose tissue and highly abundant in human plasma that had previously been identified as intelectin, a new type of $\mathrm{Ca}^{2+}$-dependent lectin with affinity to galactofuranosyl residues (the last are constituents of pathogens and dominant immunogens) [112]. So, it was suggested that a biological function of omentin/intelectin was the specific recognition of pathogens and bacterial components, playing an important role in the innate immune response to parasite infection [113]. Moreover, several studies have shown that omentin gene expression is altered by inflammatory states and obesity [114]. Indeed, Kuperman et al. have found increased gene expression of omentin in airway epithelial cells of patients with asthma [115]. Intriguingly, a differential expression of omentin mRNA occurs in omental adipose tissue of patients with Crohn's disease, suggesting that omentin could be a new candidate factor potentially involved in chronic inflammatory diseases in humans [112].

Recently, Senolt et al. have found increased levels of vaspin and reduced levels of omentin in the synovial fluid of patients with RA compared with those with OA [116]. This finding suggests that these two adipokines are likely involved in OA pathophysiology.

\section{CONCLUSIONS}

The physiological role of adipokines is becoming much more clear and several clinical and experimental lines of evidence showed their contributions to inflammatory and rheumatic disorders. The complexity of the adipokine network in the pathogenesis and progression of rheumatic diseases raises, since the beginning, one important question of whether it may be possible to target the mechanism(s) by which adipokines contribute to disease selectively without suppressing their physiological actions. The data presented in this paper suggest that adipokines and their signalling pathways may represent innovative therapeutic strategies for autoimmune and rheumatic disorders (See Supplementary Tables S1 and S2). (See Supplementary Materials available at doi:10.1100/2011/290142).

Although, these data are almost incomplete to allow translation of these approaches to clinical practice, several potential approaches are likely feasible. For instance, the control of leptin levels by using antibodies in a similar way to anti-TNF therapy might be an interesting strategy. Only further insights that clarify the mechanisms by which adipokines are regulated and which are the concrete roles of them in the rheumatic pathology could propose new pharmacological approaches for this disease. 


\section{CONFLICT OF INTERESTS}

The authors declare no competing interests.

\section{ACKNOWLEDGMENTS}

Part of the research described in this paper was supported by the Spanish Ministry of Health through the Fondo de Investigación Sanitaria, Instituto de Salud Carlos III, and by the Xunta de Galicia. The work of O. Gualillo and F. Lago is funded by the Instituto de Salud Carlos III and the Xunta de Galicia (SERGAS) through a research staff stabilization contract.

\section{REFERENCES}

[1] G. Fantuzzi, "Adipose tissue, adipokines, and inflammation," Journal of Allergy and Clinical Immunology, vol. 115, no. 5, pp. 911-920, 2005.

[2] E. Yusuf, R. G. Nelissen, A. Ioan-Facsinay et al., "Association between weight or body mass index and hand osteoarthritis: a systematic review," Annals of the Rheumatic Diseases, vol. 69, no. 4, pp. 761-765, 2010.

[3] Y. Zhang, R. Proenca, M. Maffei, M. Barone, L. Leopold, and J. M. Friedman, "Positional cloning of the mouse obese gene and its human homologue," Nature, vol. 372, no. 6505, pp. 425-432, 1994.

[4] H. Dumond, N. Presle, B. Terlain et al., "Evidence for a key role of leptin in osteoarthritis," Arthritis and Rheumatism, vol. 48, no. 11, pp. 3118-3129, 2003.

[5] M. Otero, J. J. G. Reino, and O. Gualillo, "Synergistic induction of nitric oxide synthase type II: in vitro effect of leptin and interferon-gamma in human chondrocytes and ATDC5 chondrogenic cells," Arthritis and Rheumatism, vol. 48, no. 2, pp. 404-409, 2003.

[6] M. Otero, R. Lago, F. Lago, J. J. Reino, and O. Gualillo, "Signalling pathway involved in nitric oxide synthase type II activation in chondrocytes: synergistic effect of leptin with interleukin-1," Arthritis Research \& Therapy, vol. 7, no. 3, pp. R581-R591, 2005.

[7] M. Otero, R. Lago, R. Gomez, F. Lago, J. J. Gomez-Reino, and O. Gualillo, "Phosphatidylinositol 3-kinase, MEK-1 and p38 mediate leptin/interferon-gamma synergistic NOS type II induction in chondrocytes," Life Sciences, vol. 81, no. 19-20, pp. 1452-1460, 2007.

[8] E. Toussirot, G. Streit, and D. Wendling, "The contribution of adipose tissue and adipokines to inflammation in joint diseases," Current Medicinal Chemistry, vol. 14, no. 10, pp. 1095-1100, 2007.

[9] A. Koskinen, K. Vuolteenaho, R. Nieminen, T. Moilanen, and E. Moilanen, "Leptin enhances MMP-1, MMP-3 and MMP-13 production in human osteoarthritic cartilage and correlates with MMP-1 and MMP-3 in synovial fluid from oa patients," Clinical and Experimental Rheumatology, vol. 29, no. 1, pp. 57-64, 2011.

[10] R. Gomez, M. Scotece, J. Conde, J. J. Gomez-Reino, F. Lago, and O. Gualillo, "Adiponectin and leptin increase IL-8 production in human chondrocytes," Annals of the Rheumatic Diseases, vol. 70, pp. 2052-2054, 2011.

[11] J. P. Bao, W. P. Chen, J. Feng, P. F. Hu, Z. L. Shi, and L. D. Wu, "Leptin plays a catabolic role on articular cartilage," Molecular Biology Reports, vol. 37, no. 7, pp. 3265-3272, 2010.

[12] M. S. Mutabaruka, M. A. Aissa, A. Delalandre, M. Lavigne, and D. Lajeunesse, "Local leptin production in osteoarthritis subchondral osteoblasts may be responsible for their abnormal phenotypic expression," Arthritis Research \& Therapy, vol. 12, no. 1, p. R20, 2010.

[13] T. M. Griffin, J. L. Huebner, V. B. Kraus, and F. Guilak, "Extreme obesity due to impaired leptin signaling in mice does not cause knee osteoarthritis," Arthritis and Rheumatism, vol. 60, no. 10, pp. 2935-2944, 2009.

[14] M. Otero, R. Logo, R. Gomez et al., "Changes in plasma levels of fat-derived hormones adiponectin, leptin, resistin and visfatin in patients with rheumatoid arthritis," Annals of the Rheumatic Diseases, vol. 65, no. 9, pp. 1198-1201, 2006.

[15] D. A. Fraser, J. Thoen, J. E. Reseland, O. Forre, and J. Kjeldsen-Kragh, "Decreased CD4+ lymphocyte activation and increased interleukin-4 production in peripheral blood of rheumatoid arthritis patients after acute starvation," Clinical Rheumatology, vol. 18, no. 5, pp. 394-401, 1999.

[16] E. Bernotiene, G. Palmer, and C. Gabay, "The role of leptin in innate and adaptive immune responses," Arthritis Research and Therapy, vol. 8, no. 5, article 217, 2006. 
[17] S. W. Lee, M. C. Park, Y. B. Park, and S. K. Lee, "Measurement of the serum leptin level could assist disease activity monitoring in rheumatoid arthritis," Rheumatology International, vol. 27, no. 6, pp. 537-540, 2007.

[18] B. Targonska-Stepniak, M. Majdan, and M. Dryglewska, "Leptin serum levels in rheumatoid arthritis patients: relation to disease duration and activity," Rheumatology International, vol. 28, no. 6, pp. 585-591, 2008.

[19] S. M. Olama, M. K. Senna, and M. Elarman, "Synovial/Serum leptin ratio in rheumatoid arthritis: the association with activity and erosion," Rheumatology International. In press.

[20] Q. L. Lam and L. Lu, "Role of leptin in immunity," Cellular and Molecular Immunology, vol. 4, pp. 1-13, 2007.

[21] V. De Rosa, C. Procaccini, G. Cali et al., "A key role of leptin in the control of regulatory T cell proliferation," Immunity, vol. 26, no. 2, pp. 241-255, 2007.

[22] C. Procaccini, V. De Rosa, M. Galgani et al., "An oscillatory switch in mTOR kinase activity sets regulatory T cell responsiveness," Immunity, vol. 33, no. 6, pp. 929-941, 2010.

[23] G. Palmer, M. Aurrand-Lions, E. Contassot et al., "Indirect effects of leptin receptor deficiency on lymphocyte populations and immune response in db/db mice," Journal of Immunology, vol. 177, no. 5, pp. 2899-2907, 2006.

[24] M. McMahon, B. J. Skaggs, L. Sahakian et al., "High plasma leptin levels confer increased risk of atherosclerosis in women with systemic lupus erythematosus, and are associated with inflammatory oxidised lipids," Annals of the Rheumatic Diseases, vol. 70, no. 9, pp. 1619-1624, 2011.

[25] C. P. Chung, A. G. Long, J. F. Solus et al., "Adipocytokines in systemic lupus erythematosus: relationship to inflammation, insulin resistance and coronary atherosclerosis," Lupus, vol. 18, no. 9, pp. 799-806, 2009.

[26] T. Kadowaki and T. Yamauchi, "Adiponectin and adiponectin receptors," Endocrine Reviews, vol. 26, no. 3, pp. 439-451, 2005.

[27] D. K. Oh, T. Ciaraldi, and R. R. Henry, "Adiponectin in health and disease," Diabetes, Obesity and Metabolism, vol. 9, no. 3, pp. 282-289, 2007.

[28] J. P. Whitehead, A. A. Richards, I. J. Hickman, G. A. Macdonald, and J. B. Prins, "Adiponectin—a key adipokine in the metabolic syndrome," Diabetes, Obesity and Metabolism, vol. 8, no. 3, pp. 264-280, 2006.

[29] N. Maeda, M. Takahashi, T. Funahashi et al., "PPARgamma ligands increase expression and plasma concentrations of adiponectin, an adipose-derived protein," Diabetes, vol. 50, no. 9, pp. 2094-2099, 2001.

[30] T. Yamauchi, Y. Nio, T. Maki et al., "Targeted disruption of AdipoR1 and AdipoR2 causes abrogation of adiponectin binding and metabolic actions," Nature Medicine, vol. 13, no. 3, pp. 332-339, 2007.

[31] Y. Matsuzawa, "Therapy insight: adipocytokines in metabolic syndrome and related cardiovascular disease," Nature Clinical Practice Cardiovascular Medicine, vol. 3, no. 1, pp. 35-42, 2006.

[32] K. Ebina, A. Fukuhara, W. Ando et al., "Serum adiponectin concentrations correlate with severity of rheumatoid arthritis evaluated by extent of joint destruction," Clinical Rheumatology, vol. 28, no. 4, pp. 445-451, 2009.

[33] J. T. Giles, M. Allison, C. O. Bingham III, W. M. Scott Jr., and J. M. Bathon, "Adiponectin is a mediator of the inverse association of adiposity with radiographic damage in rheumatoid arthritis," Arthritis Care and Research, vol. 61, no. 9, pp. 1248-1256, 2009.

[34] A. Ehling, A. Schaffler, H. Herfarth et al., "The potential of adiponectin in driving arthritis," Journal of Immunology, vol. 176, no. 7, pp. 4468-4478, 2006.

[35] M. Katano, K. Okamoto, M. Arito et al., "Implication of granulocyte-macrophage colony-stimulating factor induced neutrophil gelatinase-associated lipocalin in pathogenesis of rheumatoid arthritis revealed by proteome analysis," Arthritis Research and Therapy, vol. 11, no. 1, article R3, 2009.

[36] H. M. Choi, Y. A. Lee, S. H. Lee et al., "Adiponectin may contribute to synovitis and joint destruction in rheumatoid arthritis by stimulating vascular endothelial growth factor, matrix metalloproteinase-1, and matrix metalloproteinase-13 expression in fibroblast-like synoviocytes more than proinflammatory mediators," Arthritis Research \& Therapy, vol. 11, no. 6, p. R161, 2009.

[37] N. Kusunoki, K. Kitahara, F. Kojima et al., "Adiponectin stimulates prostaglandin E(2) production in rheumatoid arthritis synovial fibroblasts," Arthritis and Rheumatism, vol. 62, no. 6, pp. 1641-1649, 2010.

[38] K. W. Frommer, B. Zimmermann, F. M. P. Meier et al., "Adiponectin-mediated changes in effector cells involved in the pathophysiology of rheumatoid arthritis," Arthritis and Rheumatism, vol. 62, no. 10, pp. 2886-2899, 2010.

[39] R. Lago, R. Gomez, M. Otero et al., "A new player in cartilage homeostasis: adiponectin induces nitric oxide synthase type II and pro-inflammatory cytokines in chondrocytes," Osteoarthritis and Cartilage, vol. 16, no. 9, pp. 1101-1109, 2008.

[40] E. H. Kang, Y. J. Lee, T. K. Kim et al., "Adiponectin is a potential catabolic mediator in osteoarthritis cartilage," Arthritis Research \& Therapy, p. R231, 2010. 
[41] I. R. Klein-Wieringa, M. Kloppenburg, Y. M. Bastiaansen-Jenniskens et al., "The infrapatellar fat pad of patients with osteoarthritis has an inflammatory phenotype," Annals of the Rheumatic Diseases, vol. 70, no. 5, pp. 851857, 2011.

[42] T. Ushiyama, T. Chano, K. Inoue, and Y. Matsusue, "Cytokine production in the infrapatellar fat pad: another source of cytokines in knee synovial fluids," Annals of the Rheumatic Diseases, vol. 62, no. 2, pp. 108-112, 2003.

[43] E. Distel, T. Cadoudal, S. Durant, A. Poignard, X. Chevalier, and C. Benelli, “The infrapatellar fat pad in knee osteoarthritis: an important source of interleukin-6 and its soluble receptor," Arthritis and Rheumatism, vol. 60, no. 11, pp. 3374-3377, 2009.

[44] T. B. Laurberg, J. Frystyk, T. Ellingsen et al., "Plasma adiponectin in patients with active, early, and chronic rheumatoid arthritis who are steroid- and disease-modifying antirheumatic drug-naive compared with patients with osteoarthritis and controls," Journal of Rheumatology, vol. 36, no. 9, pp. 1885-1891, 2009.

[45] M. Filkova, M. Liskova, H. Hulejova et al., "Increased serum adiponectin levels in female patients with erosive compared with non-erosive osteoarthritis," Annals of the Rheumatic Diseases, vol. 68, no. 2, pp. 295-296, 2009.

[46] R. Gandhi, M. Takahashi, K. Syed, J. R. Davey, and N. N. Mahomed, "Relationship between body habitus and joint leptin levels in a knee osteoarthritis population," Journal of Orthopaedic Research, vol. 28, no. 3, pp. 329-333, 2010.

[47] S. Honsawek and M. Chayanupatkul, "Correlation of plasma and synovial fluid adiponectin with knee osteoarthritis severity," Archives of Medical Research, vol. 41, no. 8, pp. 593-598, 2010.

[48] D. Hao, M. Li, Z. Wu, Y. Duan, D. Li, and G. Qiu, "Synovial fluid level of adiponectin correlated with levels of aggrecan degradation markers in osteoarthritis," Rheumatology International. In press.

[49] J. B. De Sanctis, M. Zabaleta, N. E. Bianco, J. V. Garmendia, and L. Rivas, "Serum adipokine levels in patients with systemic lupus erythematosus," Autoimmunity, vol. 42, no. 4, pp. 272-274, 2009.

[50] K. E. Sada, Y. Yamasaki, M. Maruyama et al., "Altered levels of adipocytokines in association with insulin resistance in patients with systemic lupus erythematosus," Journal of Rheumatology, vol. 33, no. 8, pp. 1545$1552,2006$.

[51] B. H. Rovin, H. Song, L. A. Hebert et al., "Plasma, urine, and renal expression of adiponectin in human systemic lupus erythematosus," Kidney International, vol. 68, no. 4, pp. 1825-1833, 2005.

[52] T. Aprahamian, R. G. Bonegio, C. Richez et al., "The peroxisome proliferator-activated receptor gamma agonist rosiglitazone ameliorates murine lupus by induction of adiponectin," Journal of Immunology, vol. 182, no. 1, pp. 340-346, 2009.

[53] M. Vadacca, D. Margiotta, A. Rigon et al., "Adipokines and systemic lupus erythematosus: relationship with metabolic syndrome and cardiovascular disease risk factors," Journal of Rheumatology, vol. 36, no. 2, pp. 295297, 2009.

[54] C. M. Steppan, S. T. Bailey, S. Bhat et al., "The hormone resistin links obesity to diabetes," Nature, vol. 409, no. 6818 , pp. 307-312, 2001.

[55] M. Degawa-Yamauchi, J. E. Bovenkerk, B. E. Juliar et al., "Serum resistin (FIZZ3) protein is increased in obese humans," Journal of Clinical Endocrinology and Metabolism, vol. 88, no. 11, pp. 5452-5455, 2003.

[56] P. G. McTernan, C. L. McTernan, R. Chetty et al., "Increased resistin gene and protein expression in human abdominal adipose tissue," Journal of Clinical Endocrinology and Metabolism, vol. 87, no. 5, pp. 2407-2410, 2002.

[57] A. Schaffler, A. Ehling, E. Neumann et al., "in synovial fluid," Journal of the American Medical Association, vol. 290, no. 13, pp. 1709-1710, 2003.

[58] M. Bokarewa, I. Nagaev, L. Dahlberg, U. Smith, and A. Tarkowski, "Resistin, an adipokine with potent proinflammatory properties," Journal of Immunology, vol. 174, no. 9, pp. 5789-5795, 2005.

[59] L. Senolt, D. Housa, Z. Vernerova et al., "Resistin in rheumatoid arthritis synovial tissue, synovial fluid and serum," Annals of the Rheumatic Diseases, vol. 66, no. 4, pp. 458-463, 2007.

[60] M. A. Gonzalez-Gay, M. T. Garcia-Unzueta, C. Gonzalez-Juanatey et al., "Anti-TNF-alpha therapy modulates resistin in patients with rheumatoid arthritis," Clinical and Experimental Rheumatology, vol. 26, no. 2, pp. 311-316, 2008.

[61] H. Forsblad d'Elia, R. Pullerits, H. Carlsten, and M. Bokarewa, "Resistin in serum is associated with higher levels of IL-1Ra in post-menopausal women with rheumatoid arthritis," Rheumatology, vol. 47, no. 7, pp. 10821087, 2008. 
[62] J. H. Lee, T. Ort, K. Ma et al., "Resistin is elevated following traumatic joint injury and causes matrix degradation and release of inflammatory cytokines from articular cartilage in vitro," Osteoarthritis and Cartilage, vol. 17, no. 5, pp. 613-620, 2009.

[63] P. A. Berry, S. W. Jones, F. M. Cicuttini, A. E. Wluka, and R. A. MacIewicz, "Temporal relationship between serum adipokines, biomarkers of bone and cartilage turnover, and cartilage volume loss in a population with clinical knee osteoarthritis," Arthritis and Rheumatism, vol. 63, no. 3, pp. 700-707, 2011.

[64] Z. Zhang, X. Xing, G. Hensley et al., "Resistin induces expression of proinflammatory cytokines and chemokines in human articular chondrocytes via transcription and messenger RNA stabilization," Arthritis and Rheumatism, vol. 62, no. 7, pp. 1993-2003, 2010.

[65] K. Almehed, H. F. d'Elia, M. Bokarewa, and H. Carlsten, "Role of resistin as a marker of inflammation in systemic lupus erythematosus," Arthritis Research and Therapy, vol. 10, no. 1, article R15, 2008.

[66] B. Samal, Y. Sun, G. Stearns, C. Xie, S. Suggs, and I. McNiece, "Cloning and characterization of the cDNA encoding a novel human pre-B-cell colony-enhancing factor," Molecular and Cellular Biology, vol. 14, no. 2, pp. 1431-1437, 1994.

[67] A. Fukuhara, M. Matsuda, M. Nishizawa et al., "Visfatin: a protein secreted by visceral fat that Mimics the effects of insulin," Science, vol. 307, no. 5708, pp. 426-430, 2005.

[68] A. Fukuhara, M. Matsuda, M. Nishizawa et al., "Retraction," Science, vol. 318, no. 5850, p. 565, 2007.

[69] D. Friebe, M. Neef, J. Kratzsch et al., "Leucocytes are a major source of circulating nicotinamide phosphoribosyltransferase (NAMPT)/pre-B cell colony (PBEF)/visfatin linking obesity and inflammation in humans," Diabetologia, vol. 54, pp. 1200-1211, 2011.

[70] V. Catalan, J. Gomez-Ambrosi, A. Rodriguez et al., "Association of increased Visfatin/PBEF/NAMPT circulating concentrations and gene expression levels in peripheral blood cells with lipid metabolism and fatty liver in human morbid obesity," Nutrition, Metabolism and Cardiovascular Diseases, vol. 21, no. 4, pp. 245253, 2011.

[71] C. A. Curat, V. Wegner, C. Sengenes et al., "Macrophages in human visceral adipose tissue: increased accumulation in obesity and a source of resistin and visfatin," Diabetologia, vol. 49, no. 4, pp. 744-747, 2006.

[72] Y. Li, Y. Zhang, B. Dorweiler et al., "Extracellular nampt promotes macrophage survival via a nonenzymatic interleukin-6/STAT3 signaling mechanism," Journal of Biological Chemistry, vol. 283, no. 50, pp. 34833$34843,2008$.

[73] Y. H. Rho, J. Solus, T. Sokka et al., "Adipocytokines are associated with radiographic joint damage in rheumatoid arthritis," Arthritis and Rheumatism, vol. 60, no. 7, pp. 1906-1914, 2009.

[74] F. Brentano, O. Schorr, C. Ospelt et al., "Pre-B cell colony-enhancing factor/visfatin, a new marker of inflammation in rheumatoid arthritis with proinflammatory and matrix-degrading activities," Arthritis and Rheumatism, vol. 56, no. 9, pp. 2829-2839, 2007.

[75] N. Busso, M. Karababa, M. Nobile et al., "Pharmacological inhibition of nicotinamide phosphoribosyltransferase/visfatin enzymatic activity identifies a new inflammatory pathway linked to NAD," PLOS ONE, vol. 3, no. 5, Article ID e2267, 2008.

[76] M. Gosset, F. Berenbaum, C. Salvat et al., "Crucial role of visfatin/pre-B cell colony-enhancing factor in matrix degradation and prostaglandin E2 synthesis in chondrocytes: possible influence on osteoarthritis," Arthritis and Rheumatism, vol. 58, no. 5, pp. 1399-1409, 2008.

[77] Y. Duan, D. Hao, M. Li et al., "Increased synovial fluid visfatin is positively linked to cartilage degradation biomarkers in osteoarthritis," Rheumatology International. In press.

[78] V. Wittamer, J. D. Franssen, M. Vulcano et al., "Specific recruitment of antigen-presenting cells by chemerin, a novel processed ligand from human inflammatory fluids," Journal of Experimental Medicine, vol. 198, no. 7, pp. 977-985, 2003.

[79] B. A. Zabel, S. J. Allen, P. Kulig et al., "Chemerin activation by serine proteases of the coagulation, fibrinolytic, and inflammatory cascades," Journal of Biological Chemistry, vol. 280, no. 41, pp. 34661-34666, 2005.

[80] K. Bozaoglu, K. Bolton, J. McMillan et al., "Chemerin is a novel adipokine associated with obesity and metabolic syndrome,” Endocrinology, vol. 148, no. 10, pp. 4687-4694, 2007.

[81] S. Luangsay, V. Wittamer, B. Bondue et al., "Mouse ChemR23 is expressed in dendritic cell subsets and macrophages, and mediates an anti-inflammatory activity of chemerin in a lung disease model," Journal of Immunology, vol. 183, no. 10, pp. 6489-6499, 2009. 
[82] J. Kaur, R. Adya, B. K. Tan, J. Chen, and H. S. Randeva, "Identification of chemerin receptor (ChemR23) in human endothelial cells: chemerin-induced endothelial angiogenesis," Biochemical and Biophysical Research Communications, vol. 391, no. 4, pp. 1762-1768, 2010.

[83] V. Berg, B. Sveinbjornsson, S. Bendiksen, J. Brox, K. Meknas, and Y. Figenschau, "Human articular chondrocytes express ChemR23 and chemerin; ChemR23 promotes inflammatory signalling upon binding the ligand chemerin(21-157)," Arthritis Research \& Therapy, vol. 12, article R228, 2010.

[84] J. Conde, R. Gomez, G. Bianco et al., "Expanding the adipokine network in cartilage: identification and regulation of novel factors in human and murine chondrocytes," Annals of the Rheumatic Diseases, vol. 70, pp. 551-559, 2011.

[85] W. Vermi, E. Riboldi, V. Wittamer et al., "Role of ChemR23 in directing the migration of myeloid and plasmacytoid dendritic cells to lymphoid organs and inflamed skin," Journal of Experimental Medicine, vol. 201, no. 4, pp. 509-515, 2005.

[86] G. De Palma, G. Castellano, A. Del Prete et al., "The possible role of ChemR23/Chemerin axis in the recruitment of dendritic cells in lupus nephritis," Kidney International, vol. 79, no. 11, pp. 1228-1235, 2011.

[87] J. J. Kanalas and U. Hopfer, "Effect of TGF-beta 1 and TNF-alpha on the plasminogen system of rat proximal tubular epithelial cells," Journal of the American Society of Nephrology, vol. 8, no. 2, pp. 184-192, 1997.

[88] B. A. Zabel, S. J. Allen, P. Kulig et al., "Chemerin activation by serine proteases of the coagulation, fibrinolytic, and inflammatory cascades," Journal of Biological Chemistry, vol. 280, no. 41, pp. 34661-34666, 2005.

[89] S. Triebel, J. Blaser, H. Reinke, and H. Tschesche, "A $25 \mathrm{kDa}$ alpha 2-microglobulin-related protein is a component of the $125 \mathrm{kDa}$ form of human gelatinase," FEBS Letters, vol. 314, no. 3, pp. 386-388, 1992.

[90] L. R. Devireddy, J. G. Teodoro, F. A. Richard, and M. R. Green, "Induction of apoptosis by a secreted lipocalin that is transcriptionally regulated by IL-3 deprivation," Science, vol. 293, no. 5531, pp. 829-834, 2001.

[91] S. T. Chu, H. J. Lin, H. L. Huang, and Y. H. Chen, "The hydrophobic pocket of 24p3 protein from mouse uterine luminal fluid: fatty acid and retinol binding activity and predicted structural similarity to lipocalins," Journal of Peptide Research, vol. 52, no. 5, pp. 390-397, 1998.

[92] J. B. Cowland and N. Borregaard, "Molecular characterization and pattern of tissue expression of the gene for neutrophil gelatinase-associated lipocalin from humans," Genomics, vol. 45, no. 1, pp. 17-23, 1997.

[93] H. C. Owen, S. J. Roberts, S. F. Ahmed, and C. Farquharson, "Dexamethasone-induced expression of the glucocorticoid response gene lipocalin 2 in chondrocytes," American Journal of Physiology-Endocrinology and Metabolism, vol. 294, no. 6, pp. E1023-E1034, 2008.

[94] K. Gupta, M. Shukla, J. B. Cowland, C. J. Malemud, and T. M. Haqqi, "Neutrophil gelatinase-associated lipocalin is expressed in osteoarthritis and forms a complex with matrix metalloproteinase 9," Arthritis and Rheumatism, vol. 56, no. 10, pp. 3326-3335, 2007.

[95] C. H. Hinze, M. Suzuki, M. Klein-Gitelman et al., "Neutrophil gelatinase-associated lipocalin is a predictor of the course of global and renal childhood-onset systemic lupus erythematosus disease activity," Arthritis and Rheumatism, vol. 60, no. 9, pp. 2772-2781, 2009.

[96] K. I. Yamamoto and S. Migita, "Complete primary structures of two major murine serum amyloid A proteins deduced from cDNA sequences," Proceedings of the National Academy of Sciences of the United States of America, vol. 82, no. 9, pp. 2915-2919, 1985.

[97] C. A. Lowell, R. S. Stearman, and J. F. Morrow, "Transcriptional regulation of serum amyloid A gene expression," Journal of Biological Chemistry, vol. 261, no. 18, pp. 8453-8461, 1986.

[98] M. C. de Beer, C. M. Beach, S. I. Shedlofsky, and F. C. de Beer, "Identification of a novel serum amyloid A protein in BALB/c mice," Biochemical Journal, vol. 280, part 1, pp. 45-49, 1991.

[99] B. Kluve-Beckerman, M. L. Drumm, and M. D. Benson, "Nonexpression of the human serum amyloid A three (SAA3) gene," DNA and Cell Biology, vol. 10, no. 9, pp. 651-661, 1991.

[100] B. Kluve-Beckerman and M. Song, "Genes encoding human serum amyloid A proteins SAA1 and SAA2 are located $18 \mathrm{~kb}$ apart in opposite transcriptional orientations," Gene, vol. 159, no. 2, pp. 289-290, 1995.

[101] C. S. Reigstad, G. O. Lunden, J. Felin, and F. Backhed, "Regulation of serum amyloid A3 (SAA3) in mouse colonic epithelium and adipose tissue by the intestinal microbiota," PLoS One, vol. 4, Article ID e5842, 2009.

[102] M. Frasshauer, J. Klein, S. Kralisch et al., "Serum amyloid A3 expression is stimulated by dexamethasone and interleukin-6 in 3T3-L1 adipocytes," Journal of Endocrinology, vol. 183, no. 3, pp. 561-567, 2004. 
[103] Y. Lin, M. W. Rajala, J. P. Berger, D. E. Moller, N. Barzilai, and P. E. Scherer, "Hyperglycemia-induced production of acute phase reactants in adipose tissue," Journal of Biological Chemistry, vol. 276, no. 45, pp. 42077-42083, 2001.

[104] J. C. Pickup, M. B. Mattock, G. D. Chusney, and D. Burt, "NIDDM as a disease of the innate immune system: association of acute- phase reactants and interleukin-6 with metabolic syndrome X," Diabetologia, vol. 40, no. 11, pp. 1286-1292, 1997.

[105] C. Y. Han, S. Subramanian, C. K. Chan et al., "Adipocyte-derived serum amyloid A3 and hyaluronan play a role in monocyte recruitment and adhesion," Diabetes, vol. 56, no. 9, pp. 2260-2273, 2007.

[106] G. Sommer, S. Weise, S. Kralisch et al., "The adipokine SAA3 is induced by interleukin-1beta in mouse adipocytes," Journal of Cellular Biochemistry, vol. 104, no. 6, pp. 2241-2247, 2008.

[107] R. Vallon, F. Freuler, N. Desta-Tsedu et al., "Serum amyloid a (apoSAA) expression is up-regulated in rheumatoid arthritis and induces transcription of matrix metalloproteinases," Journal of Immunology, vol. 166, no. 4, pp. 2801-2807, 2001.

[108] K. Tatemoto, M. Hosoya, Y. Habata et al., "Isolation and characterization of a novel endogenous peptide ligand for the human APJ receptor," Biochemical and Biophysical Research Communications, vol. 251, no. 2, pp. 471-476, 1998.

[109] D. Daviaud, J. Boucher, S. Gesta et al., "TNFalpha up-regulates apelin expression in human and mouse adipose tissue," The FASEB Journal, vol. 20, no. 9, pp. 1528-1530, 2006.

[110] H. Xu, G. T. Barnes, Q. Yang et al., "Chronic inflammation in fat plays a crucial role in the development of obesity-related insulin resistance," Journal of Clinical Investigation, vol. 112, no. 12, pp. 1821-1830, 2003.

[111] K. Hida, J. Wada, J. Eguchi et al., "Visceral adipose tissue-derived serine protease inhibitor: A unique insulinsensitizing adipocytokine in obesity," Proceedings of the National Academy of Sciences of the United States of America, vol. 102, no. 30, pp. 10610-10615, 2005.

[112] A. Schaffler, M. Neumeier, H. Herfarth, A. Furst, J. Scholmerich, and C. Buchler, "Genomic structure of human omentin, a new adipocytokine expressed in omental adipose tissue," Biochimica et Biophysica Acta, vol. 1732, no. 1-3, pp. 96-102, 2005.

[113] L. Gerwick, G. Corley-Smith, and C. J. Bayne, "Gene transcript changes in individual rainbow trout livers following an inflammatory stimulus," Fish and Shellfish Immunology, vol. 22, no. 3, pp. 157-171, 2007.

[114] C. M. de Souza Batista, R. Z. Yang, M. J. Lee et al., "Omentin plasma levels and gene expression are decreased in obesity," Diabetes, vol. 56, no. 6, pp. 1655-1661, 2007.

[115] D. A. Kuperman, C. C. Lewis, P. G. Woodruff et al., "Dissecting asthma using focused transgenic modeling and functional genomics," Journal of Allergy and Clinical Immunology, vol. 116, no. 2, pp. 305-311, 2005.

[116] L. Senolt, M. Polanska, M. Filkova et al., "Vaspin and omentin: new adipokines differentially regulated at the site of inflammation in rheumatoid arthritis," Annals of the Rheumatic Diseases, vol. 69, no. 7, pp. 1410-1411, 2010.

\section{This article should be cited as follows:}

Morena Scotece, Javier Conde, Rodolfo Gómez, Veronica López, Francisca Lago, Juan Jesus GómezReino, and Oreste Gualillo, "Beyond Fat Mass: Exploring the Role of Adipokines in Rheumatic Diseases," TheScientificWorldJOURNAL, vol. 11, pp. 1932-1947, 2011. 


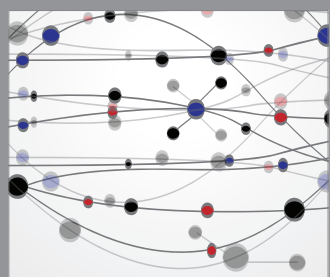

The Scientific World Journal
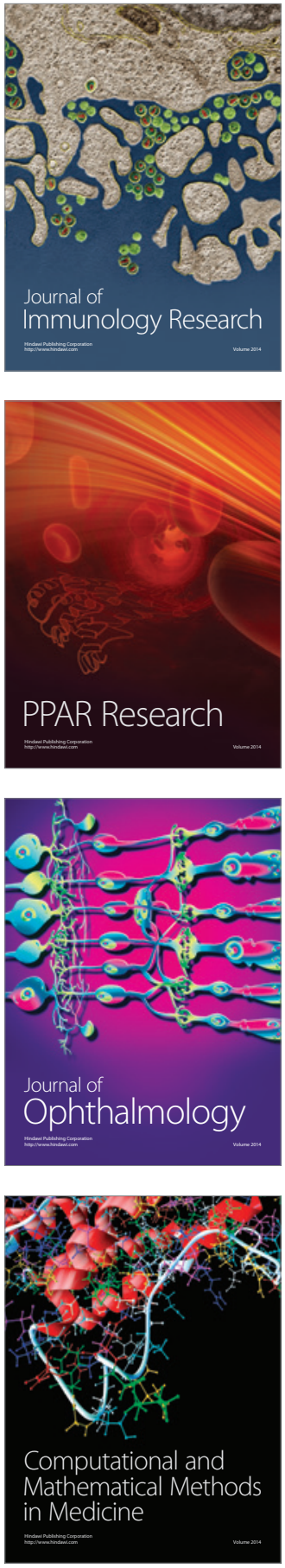

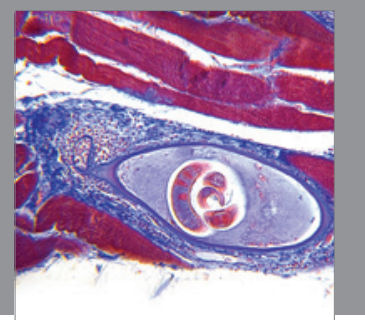

Gastroenterology

Research and Practice
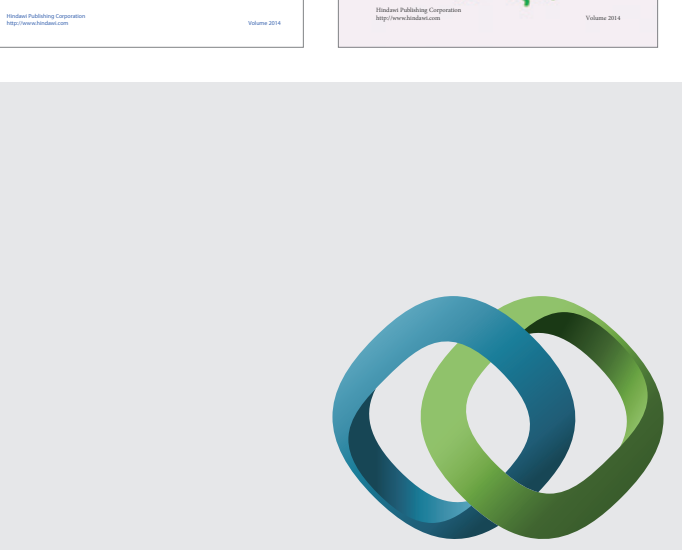

\section{Hindawi}

Submit your manuscripts at

http://www.hindawi.com
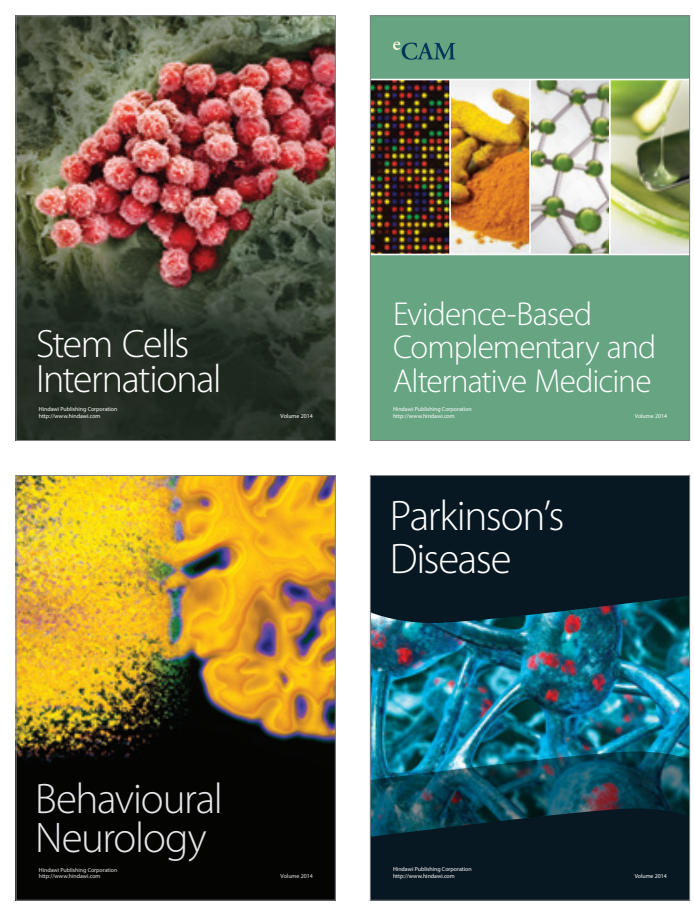

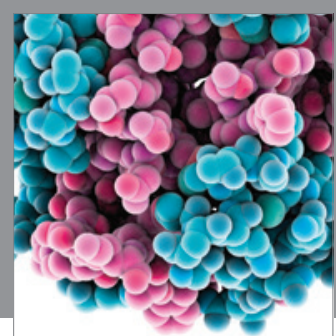

Journal of
Diabetes Research

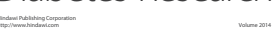

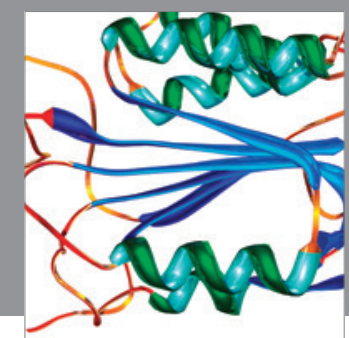

Disease Markers
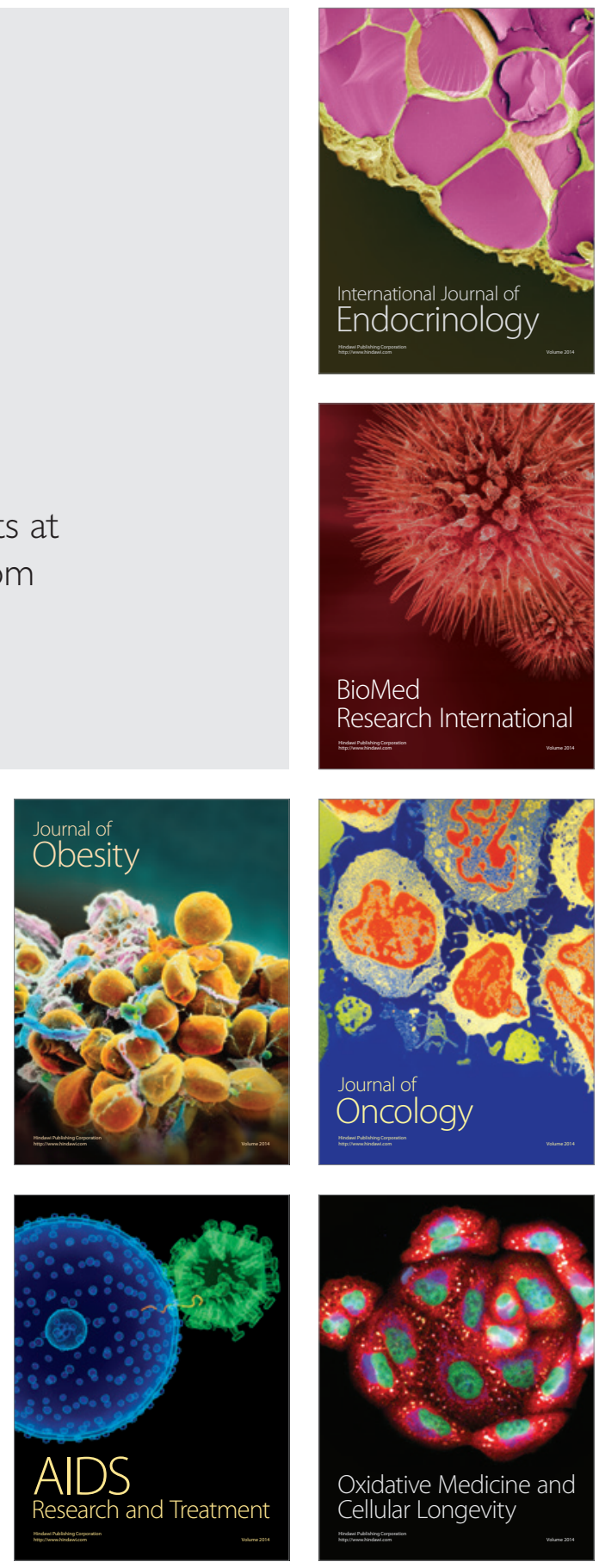